SLEEP DISORDERED BREATHING

\title{
Craniofacial profile in Asian and white subjects with obstructive sleep apnoea
}

\section{B Lam, M S M Ip, E Tench, C F Ryan}

Thorax 2005;60:504-510. doi: 10.1136/thx.2004.031591

See end of article for authors' affiliations

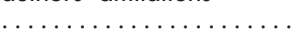

Correspondence to:

Dr C F Ryan, UBC

Respiratory Clinic,

Vancouver General

Hospital, 2775 Heather

Street, Vancouver, BC,

V5Z 3J5, Canada; fryan@

interchange.ubc.ca

Received 13 July 2004

Accepted

24 February 2005

\begin{abstract}
Background: Clinical detection of structural narrowing of the upper airway may facilitate early recognition of obstructive sleep apnoea (OSA). To determine whether the craniofacial profile predicts the presence of OSA, the upper airway and craniofacial structure of 239 consecutive patients (164 Asian and 75 white subjects) referred to two sleep centres (Hong Kong and Vancouver) were prospectively examined for suspected sleep disordered breathing.

Methods: All subjects underwent a history and physical examination with measurements of anthropometric parameters and craniofacial structure including neck circumference, thyromental distance, thyromental angle, and Mallampati oropharyngeal score. OSA was defined as an apnoea-hypopnoea index (AHI) of $\geqslant 5$ /hour on full overnight polysomnography.

Results: Discriminant function analysis indicated that the Mallampati score $(F=0.70)$, thyromental angle $(F=0.60)$, neck circumference $(F=0.54)$, body mass index $(F=0.53)$, and age $(F=0.53)$ were the best predictors of OSA. After controlling for ethnicity, body mass index and neck circumference, patients with OSA were older, had larger thyromental angles, and higher Mallampati scores than non-apnoeic subjects. These variables remained significantly different between OSA patients and controls across a range of cutoff values of AHI from 5 to $30 /$ hour.

Conclusions: A crowded posterior oropharynx and a steep thyromental plane predict OSA across two different ethnic groups and varying degrees of obesity.
\end{abstract}

S tructural narrowing of the upper airway is a major factor contributing to pharyngeal occlusion during sleep in the obstructive sleep apnoea-hypoponea syndrome (OSAHS). ${ }^{1}$ Patients with OSAHS manifest a spectrum of abnormalities of craniofacial soft tissue and skeletal anatomy that appear to act synergistically to promote upper airway obstruction during sleep. ${ }^{2}$ Adiposity in the neck region and retrognathia are recognised risk factors for OSAHS. ${ }^{3-5}$ Many patients with OSAHS have evidence of both neck adiposity and skeletal abnormalities. ${ }^{2}$ The relative contributions of soft tissue and skeletal abnormalities vary among patients and between ethnic groups: Asians have more pronounced skeletal abnormalities and are less obese than white subjects for a given degree of severity of OSAHS. ${ }^{6}$

Epidemiological data demonstrating the relationship between OSAHS and cardiovascular morbidity highlight the potential importance of early recognition and treatment. ${ }^{7}$ Yet, despite recent advances in the diagnosis and treatment of sleep disordered breathing, OSAHS remains under-recognised. This may be partly related to the limited availability of diagnostic facilities and to the non-specific nature of symptoms associated with OSAHS. Various attempts to use diagnostic algorithms based on features of the history, physical examination, and overnight oximetry to identify patients with OSAHS have met with limited success. ${ }^{89}$ Common symptoms such as snoring and daytime hypersomnolence lack specificity for the diagnosis of OSAHS. Witnessed apnoea is an important clue but requires observation by a regular bed partner. Oximetry is widely used as a case selection tool for OSA, but its sensitivity and specificity in predicting OSA are quite variable. ${ }^{8}$ Prediction models based on physical findings have used measurements that may not be easily incorporated into the routine physical examination. ${ }^{10}$ Some readily detectable visual cues to the presence of OSAHS would be useful for the primary care physician whose encounters with patients may be brief and infrequent.

In a preliminary report we observed that certain characteristics of craniofacial structure were commonplace in patients with OSAHS and could be readily identified on routine physical examination. ${ }^{11}$ We therefore hypothesised that a simple clinical assessment of craniofacial profile using measurements of soft tissue and skeletal abnormalities that may contribute to upper airway narrowing would predict the presence of OSAHS. We also wished to determine whether ethnicity affected the utility of this assessment.

Some of the results of this study have been previously reported in the form of an abstract. ${ }^{12}$ The study was reviewed and approved by the ethics review boards of our respective institutions.

\section{METHODS}

We studied 239 consecutive patients referred for the assessment of possible OSAHS to two university based regional sleep disorders clinics-one in Hong Kong and the other in Vancouver, British Columbia-between January and December 1999. At the time of the initial visit, having given written informed consent, each patient underwent a detailed history and physical examination including anthropometric measurements-height, weight, and calculation of body mass index (BMI).

\section{Craniofacial measurements}

Craniofacial measurements were made with the patient seated upright in a straight backed chair in the natural head position looking straight ahead (fig 1). Natural head position

Abbreviations: $\mathrm{AHI}$, apnoea-hypopnoea index; $\mathrm{BMI}$, body mass index; MS, Mallampati score; NC, neck circumference; OSA, obstructive sleep apnoea; OSAHS, obstructive sleep apnoea-hypoponea syndrome; TMA, thyromental angle; TMD, thyromental distance 


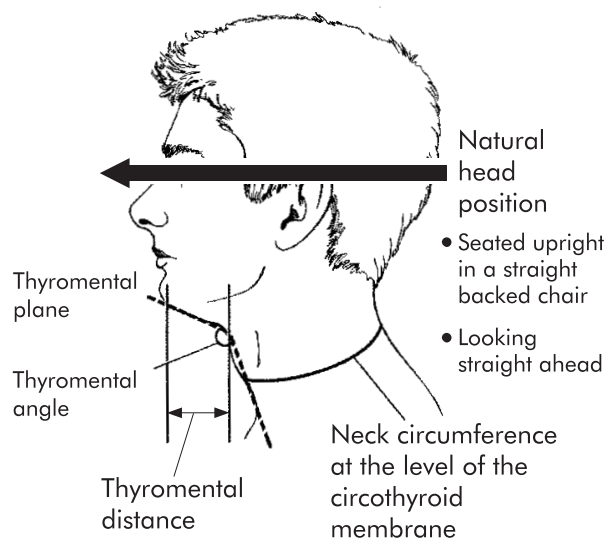

Figure 1 Profile of the head and neck region illustrating the following features: thyromental plane: running through the soft tissue mentum and the thyroid prominence; thyromental angle (TMA): between the thyromental plane and the soft tissue plane of the anterior neck; thyromental distance (TMD): the horizontal distance between the thyroid prominence and a perpendicular dropped from the soft tissue mentum; neck circumference (NC): at the level of the cricothyroid membrane.

is used increasingly as a logical reference for the evaluation of craniofacial morphology and has been shown to be stable and reproducible in the short term to within $2^{\circ} .{ }^{13}$ The following measurements were made:

- Neck circumference (NC): at the level of the cricothyroid membrane, measured with a tape measure.

- Thyromental distance (TMD): the horizontal distance from the thyroid prominence to a perpendicular dropped from the soft tissue mentum, measured with a modified tape measure with a vertical attachment.

- Thyromental angle (TMA): the angle between the soft tissue plane of the anterior neck and a plane running through the soft tissue mentum and the thyroid prominence, measured from a lateral photograph of the head and neck taken in the natural head position with the patient looking straight ahead. The camera was mounted on a tripod with the front of the lens oriented vertically, at a constant distance of 6 feet from the patients' left ear and with the height adjusted to maintain the body of the lens horizontal, centred on the tragus of the left ear.

- Mallampati oropharyngeal score (MS): ${ }^{14}$ measured during a breath hold at end-tidal inspiration with the mouth wide open, tongue maximally protruded, but without phonation or attempted elevation of the soft palate (fig 2).

\section{Reliability of measurements}

A training session was held at one centre before study commencement during which the measurement techniques for all variables were standardised. Measurements were performed by a single investigator at each centre (CFR and $\mathrm{BL}$ ). Intra-observer agreement was assessed for MS, NC, and TMD in a sample of 20 patients. On a sample of 50 randomly selected patients, tests of intra- and inter-observer agreement were performed for the measurement of TMA. For intraobserver reliability, measurements were made in random order on two occasions separated by several days. For interobserver reliability, measurements were made at one centre and repeated in random order on duplicate images at the other centre in a blinded fashion.

\section{Polysomnography}

Each patient had full overnight polysomnography within 12 weeks of the initial assessment, reflecting the waiting

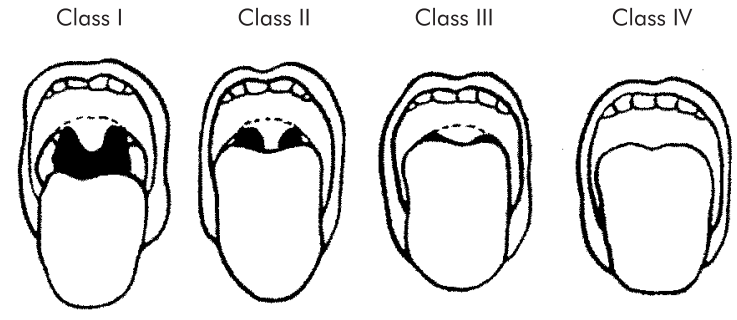

Figure 2 Mallampati oropharyngeal score (MS) measured during a breath hold at end-tidal inspiration with the mouth wide open, tongue maximally protruded, but without phonation or attempted elevation of the soft palate.

times in our facilities at the time of the study. Polysomnography was performed using methodology, instrumentation, and analysis standardised between both sleep centres according to the recommendations on syndrome definition and measurement techniques published by the American Academy of Sleep Medicine. ${ }^{15}$ Polysomnograms were scored by technicians at the respective sites according to usual clinical practice. OSA was defined as an apnoeahypopnoea index (AHI) of $\geqslant 5 /$ hour of total sleep time.

\section{Statistical analysis}

The rationale and methodology for the statistical analysis are outlined in detail in the supplement available online at the Thorax website (http://www.thoraxjnl.com/supplemental). A summary of the analysis is given here. Spearman rank correlation, to account for outlying measurements, was used to assess intra- and inter-observer reliability in the craniofacial measurements. SPSS Version 10.0.7 was used for all other analyses. The statistical strategy in this study was to determine which clinical, anthropometric, and craniofacial variables could be broadly applied to predict which patients were more likely to have OSA. The clinical variables of interest were age, BMI, NC, MS, TMA, TMD, and Epworth Sleepiness Scale. First, three Hotelling's $\mathrm{T}^{2}$ analyses examined the relationship between (1) sex, (2) ethnic group, and (3) OSA and the clinical variables. The sample of patients was then randomly divided into two groups of 120 and 119 patients balanced with respect to sex, ethnicity, and OSA status. Discriminant function analysis was applied to the initial group of 120 patients to generate an equation using the clinical variables to predict whether a patient was more likely to have OSA or to be normal. A receiver operator characteristic curve (ROC) was also plotted to assess the equation in terms of sensitivity and specificity and for comparison with other previously published prediction equations. The second group of 119 patients was used to perform a prospective data analysis (cross validation) to assess the performance of the equation in a different but comparable group from the one in which it was originally developed. Finally, an analysis of covariance (ANCOVA) was performed to effectively make all patients equivalent for BMI, NC, and ethnic group in order to determine whether the relationship between selected clinical variables from the discriminant analysis was still significant when the group of patients was statistically rendered equivalent on these three variables. Additionally, several different cut-offs for the definition of OSA (AHI $\geqslant 5, \geqslant 10$, $\geqslant 15, \geqslant 30$ ) were tested to determine whether the same variables were significant for more rigorous definitions of OSA. Summary data are presented as mean (SD).

\section{RESULTS}

Demographic data summarised by group differences in disease category, ethnic group, and sex are shown in table 1. Of the 239 patients, 201 had OSA ( $\mathrm{AHI} \geqslant 5)$. As a group, these 
Table 1 Group differences by disease category, ethnicity and sex†

\begin{tabular}{|c|c|c|c|c|c|c|}
\hline \multirow[b]{2}{*}{ Variable } & \multicolumn{2}{|c|}{ Disease category } & \multicolumn{2}{|l|}{ Ethnicity } & \multicolumn{2}{|l|}{ Sex } \\
\hline & $\begin{array}{l}\text { OSA } \\
(n=201)\end{array}$ & $\begin{array}{l}\text { Normal } \\
(n=38)\end{array}$ & $\begin{array}{l}\text { Asian } \\
(n=164)\end{array}$ & $\begin{array}{l}\text { White } \\
(n=75)\end{array}$ & $\begin{array}{l}\text { Male } \\
(n=199)\end{array}$ & $\begin{array}{l}\text { Female } \\
(n=40)\end{array}$ \\
\hline Age (years) & $49(12)$ & $44(13)$ & $49(11)$ & $47(14)$ & $49(12)$ & $48(12)$ \\
\hline$M / F$ & $169 / 32$ & $30 / 8$ & $140 / 24$ & $59 / 16$ & - & - \\
\hline $\mathrm{BMI}\left(\mathrm{kg} / \mathrm{m}^{2}\right)$ & $29(5)$ & $27(5)$ & $29(4)$ & $30(7)$ & $29(5)$ & $30(7)$ \\
\hline Neck $(\mathrm{cm})$ & $40(4)$ & $39(4)$ & 40 (3) & $41(4)$ & $41(3)$ & $36(4)^{*}$ \\
\hline$M S(1-4)$ & $3.7(0.5)$ & $3.4(0.8)^{*}$ & $3.9(0.4)$ & $3.2(0.8)^{*}$ & $3.7(0.6)$ & $3.5(0.6)$ \\
\hline TMA (degrees) & $161(18)$ & $149(19)^{*}$ & $161(18)$ & $156(19)$ & $160(18)$ & $155(22)$ \\
\hline TMD $(\mathrm{cm})$ & $5(1)$ & $5(1)$ & $5(1)$ & $6(1)^{*}$ & $5(1)$ & $5(1)$ \\
\hline ESS & $11(5)$ & $11(5)$ & $11(5)$ & $11(5)$ & $11(5)$ & $11(6)$ \\
\hline $\mathrm{AHI}(/ \mathrm{h})$ & $32(22)$ & $3(2)^{*}$ & $30(24)$ & 22 (19) & $30(23)$ & $14(13)^{*}$ \\
\hline
\end{tabular}

patients were middle aged (49 (12) years), moderately obese (BMI $29.5(6.3) \mathrm{kg} / \mathrm{m}^{2}$ ), and had moderate to severe OSA (AHI $31.9(22.2) / \mathrm{h})$. All of the Hong Kong subjects $(\mathrm{n}=154)$ and 10 of the Vancouver subjects were Asian; the remaining 75 Vancouver subjects were white. The majority (83\%) were male. There was no change in body weight between the performance of the anthropometric measurements and the polysomnogram.

Spearman rank correlation indicated intra-observer agreement (confidence intervals) of 0.81 (0.56 to 0.92) for MS; 0.97 (0.93 to 0.99 ) for NC: and 0.85 (0.71 to 0.90$)$ for TMD. For TMA, intra-observer agreement was 0.91 (0.87 to 0.94$)$ and inter-observer agreement was 0.83 (0.71 to 0.90$)$. Values greater than 0.80 indicate acceptable reliability.

\section{Hotelling's $\mathrm{T}^{2}$ analyses of OSA and clinical variables}

The results of the initial $\mathrm{T}^{2}$ analyses (OSA defined as AHI $\geqslant 5$ ) of the full demographic data set are provided in table 1 . Patients with OSA had higher MS, larger TMA, and higher AHI than subjects without OSA. Asians had higher MS and shorter TMD than whites. Men had larger NC and higher AHI than women. Although the proportion of women was somewhat greater among whites, this was not significant. Because of their small numbers, it was not possible to repeat the same analyses on the women as a subgroup. When women were excluded from the analysis, there were no differences in the overall results when comparing groups by disease category or ethnicity, or in the multivariate analyses.

\section{Discriminant analysis}

From the preceding analyses a subset of potentially useful predictor variables including TMA, MS, age, BMI, NC and TMD was selected for a discriminant analysis. This analysis confirmed that these variables could be used to discriminate

\begin{tabular}{lc}
$\begin{array}{l}\text { Table } 2 \\
\text { prediction of OSA }(\mathrm{AHI} \geqslant 5) \dagger\end{array}$ \\
\hline Variable & Function \\
\hline Mallampati score & $0.70^{*}$ \\
Thyromental angle & $0.60^{*}$ \\
Neck circumference & $0.54^{*}$ \\
Body mass index & $0.53^{*}$ \\
Age & $0.53^{*}$ \\
Thyromental distance & -0.13 \\
\hline
\end{tabular}

*Significant correlation.

tCorrelation between clinical variables and discriminant function for the initial sample of 120 patients split by OSA.
Table 3 Prediction equations for the presence or absence of OSA $(\mathrm{AHI} \geqslant 5)^{*}$

\begin{tabular}{llll}
\hline Variable & & OSA & Normal \\
\hline $\begin{array}{l}\text { Mallampati score } \\
+\end{array}$ & $\times$ & $(7.135)$ & $(6.227)$ \\
$\begin{array}{l}\text { Thyromental angle } \\
+\end{array}$ & $\times$ & $(0.171)$ & $(0.163)$ \\
$\begin{array}{l}\text { Neck circumference } \\
-\end{array}$ & $\times$ & $(2.883)$ & $(2.758)$ \\
$\begin{array}{l}\text { Body mass index } \\
+\end{array}$ & $\times$ & $(0.075)$ & $(0.163)$ \\
$\begin{array}{l}\text { Age } \\
+ \\
\begin{array}{l}\text { Thyromental distance } \\
=\end{array}\end{array}$ & $\times$ & $(0.166)$ & $(0.131)$ \\
& & $(102.571)$ & $(92.088)$ \\
& & OSA score & Normal score
\end{tabular}

*A patient's score is calculated for both equations by multiplying the raw score for each coefficient and summing these products. The larger score indicates the group to which the patient more likely belongs.

between patients with OSA and normal subjects. The size of each correlation (function) described in table 2 reveals the strength of the relationship between that predictor and OSA. Patients with OSA had, in order of importance, significantly higher MS, larger TMA, larger NC, higher BMI, and were older than normal subjects. An equation was derived from this analysis to categorise patients as either OSA or normal (table 3). When this equation was applied to the initial set of 120 patients from which it was derived, it correctly classified $86 \%$ of the patients. When applied prospectively to the second set of 119 patients its performance was comparable, correctly classifying $82 \%$ of patients as either OSA or normal.

\section{Analysis of covariance (ANCOVA)}

Following the positive results of the discriminant analysis, the data from the 120 and 119 patients were again pooled for the ANCOVA. The purpose of the ANCOVA was to examine the relationship between OSA and MS, TMA, TMD, and age after statistically removing prior differences in three potentially important confounding variables-BMI, NC, and ethnic group. By treating ethnic group as an independent variable rather than a covariate, differences between ethnic groups could also be examined separately after removing prior differences in BMI and NC. BMI was significantly related to the set of dependent variables, as was NC and ethnic group $(p<0.05)$. Thus, these three variables could be used to statistically render patients as if they had all scored equally on these variables. It is notable that there was no interaction between OSA and ethnic group. The relationship between 
Table 4 Comparisons between subjects with OSA and normal subjects for different cut off values for apnoea-hypopnoea index after adjustment for BMI, neck circumference, and ethnicity $\dagger$

\begin{tabular}{|c|c|c|c|c|c|c|c|c|c|}
\hline \multirow[b]{2}{*}{$A H I(/ h)$} & \multicolumn{3}{|c|}{ Age (years) } & \multicolumn{3}{|c|}{ Mallampati score (1-4) } & \multicolumn{3}{|c|}{ Thyromental angle (degrees) } \\
\hline & OSA & Normal & $p$ value & OSA & Normal & $p$ value & OSA & Normal & $p$ value \\
\hline $\begin{array}{l}\geqslant 5 \\
n\end{array}$ & $\begin{array}{l}49 \\
(201)\end{array}$ & $\begin{array}{l}44 \\
\text { (38) }\end{array}$ & * & 3.7 & 3.4 & * & 161 & 151 & * \\
\hline $\begin{array}{l}\geqslant 10 \\
n\end{array}$ & $\begin{array}{l}50 \\
(168)\end{array}$ & $\begin{array}{l}46 \\
\text { (71) }\end{array}$ & * & 3.7 & 3.5 & * & 161 & 154 & * \\
\hline $\begin{array}{l}\geqslant 15 \\
n\end{array}$ & $\begin{array}{l}50 \\
(143)\end{array}$ & $\begin{array}{l}46 \\
(96)\end{array}$ & * & 3.8 & 3.5 & * & 161 & 156 & * \\
\hline $\begin{array}{l}\geqslant 30 \\
n\end{array}$ & $\begin{array}{l}51 \\
(88)\end{array}$ & $\begin{array}{l}47 \\
\text { (151) }\end{array}$ & * & 3.8 & 3.6 & * & 164 & 157 & * \\
\hline
\end{tabular}

Values are means.

* $\mathrm{p}<0.05$.

†ANCOVA with adjustment for BMI, neck circumference and ethnicity; Bonferroni corrections for multiple comparisons; and follow-up univariate analyses.

OSA and age, MS, TMA and TMD could therefore be examined without regard to ethnic group; also investigation of ethnic group could proceed independently of OSA status. In addition, we were interested in determining whether the relationship remained stable using varying cut off levels of AHI to define OSA ( $\geqslant 5, \geqslant 10, \geqslant 15$, and $\geqslant 30)$.

\section{OSA}

The set of dependent variables was related significantly to OSA $(p<0.05)$. Follow up ANOVAs, with Bonferroni corrections to control the error rate, showed that age, MS, and TMA distinguished patients with OSA from normal subjects $(p<0.05)$. In order of importance, these comparisons revealed that OSA patients were older than normal subjects $(M=49.27 v 44.08)$, had larger TMA $(M=161.17 v 148.92)$ and had higher MS $(\mathrm{M}=3.71 \vee 3.37)$.

\section{Ethnic group}

After removal of prior differences in BMI and NC, Asians differed from whites in MS, TMD and TMA. In order of importance, Asians had higher MS, smaller TMD, and larger TMA than white subjects $(\mathrm{p}<0.05)$.
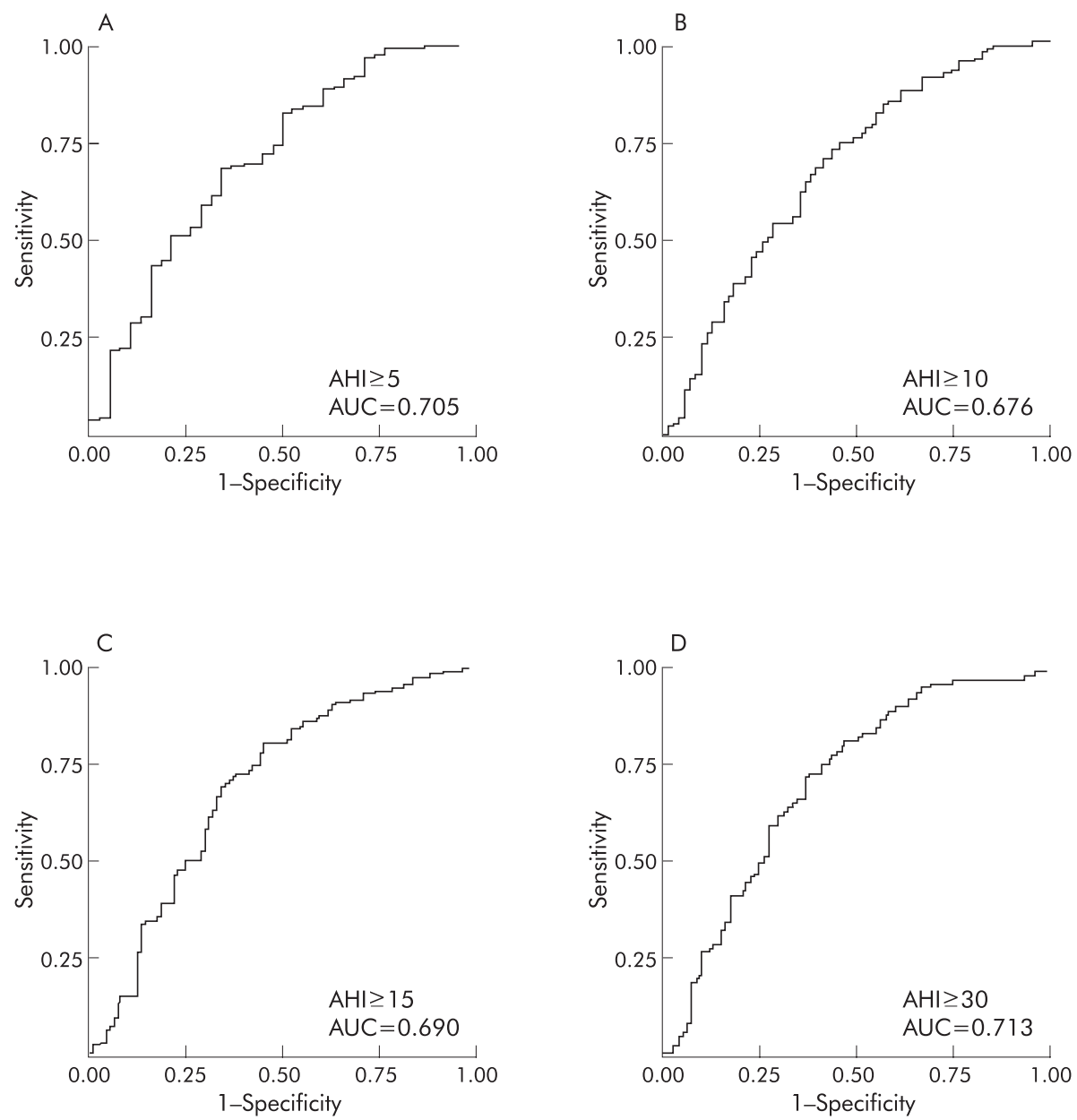

Figure 3 Receiver operator characteristic curves for the predictive equations using different cut off values of apnoea-hypopnoea index (AHI) for the definition of obstructive sleep apnoea: (A) $A H I \geqslant 5,(B) A H I \geqslant 10,(C) A H I \geqslant 15,(D) A H I \geqslant 30$. AUC = area under the curve. 
A

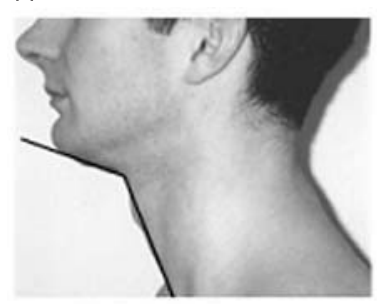

TMA $134^{\circ}$

AHI $4 / h r$

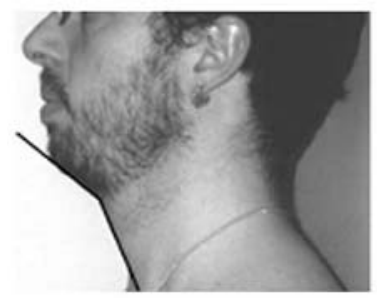

TMA $152^{\circ}$

AHI 16/hr

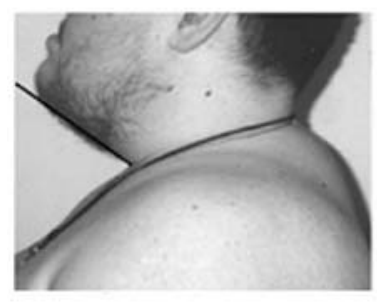

TMA $180^{\circ}$

AHI 46/hr
B

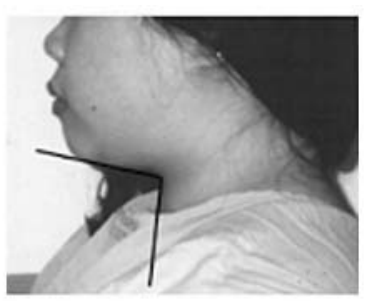

TMA $93^{\circ}$

AHI 6/hr

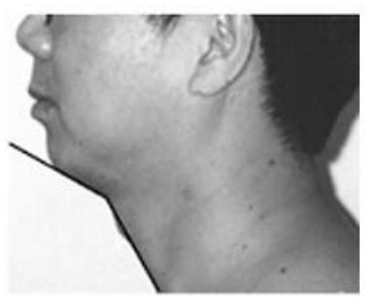

TMA $148^{\circ}$

AHI $11 / \mathrm{hr}$

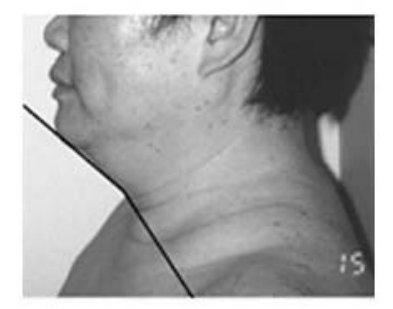

TMA $160^{\circ}$

AHI 65/hr

Figure 4 Lateral photographs of the head and neck region showing examples of the thyromental angle in (A) white and (B) Asian patients with varying degrees of severity of obstructive sleep apnoea. TMA = thyromental angle (degrees); $\mathrm{AHI}=$ apnoea-hypopnoea index (/hour).

\section{Varying definitions of OSA}

For each definition of OSA (AHI $\geqslant 5, \geqslant 10$, $\geqslant 15$, and $\geqslant 30$ ), increasing age, MS, and TMA were significantly associated with the presence of OSA (table 4). ROC curves of the predictive equations for different degrees of OSA severity are shown in fig 3 . The area under the curve (AUC) was similar for each cut off $(0.705$ for $\mathrm{AHI} \geqslant 5 ; 0.676$ for $\mathrm{AHI} \geqslant 10 ; 0.696$ for $\mathrm{AHI} \geqslant 15$; and 0.713 for $\mathrm{AHI} \geqslant 30$ ). Figure 4 shows examples of the TMA measurement in white and Asian patients with different degrees of severity of OSA.

\section{DISCUSSION}

Our results indicate that patients with OSA of differing ethnicity and varying degrees of obesity have a crowded posterior oropharynx and a steep thyromental plane compared with non-apnoeic controls. After accounting for ethnic group, BMI and NC, patients with OSA are older and have larger MS and TMA. These craniofacial features probably reflect structural narrowing of the upper airway that contributes directly to the pathogenesis of upper airway obstruction in OSA. A crowded posterior oropharynx and a steep thyromental plane appear to be particularly useful clinical markers of OSA since they remain strong predictors even after correction for two important confounding variables — ethnicity and obesity - and over a wide range of cutoff values for the definition of OSA.

Whether clinical features can be reliably used to predict OSA among suspected individuals is still controversial. ${ }^{16-18}$

Ross and co-workers ${ }^{8}$ conducted a systematic review and meta-analysis of the literature regarding the diagnosis of OSA. Among the articles reviewed were eight studies reporting prediction equations. The pooled estimates of these models were 66.5 (14)\% for sensitivity and 88.7 (4.9)\% for specificity. Rowley and colleagues prospectively evaluated four clinical prediction equations for the diagnosis of OSA and the prioritisation of patients for polysomnography. ${ }^{9}$ The performance characteristics of these equations were similar with AUCs ranging from 0.669 to 0.736 , comparable to those of the ROC curves for the prediction equation in our study. The authors concluded that these models could be used as part of a clinical pathway to prioritise patients for polysomnography.

Several craniofacial characteristics have been shown to be associated with the development and severity of sleep disordered breathing. The most commonly reported abnormalities include an inferiorly positioned hyoid bone, a posteriorly placed maxilla and mandible, an enlarged tongue and soft palate, and a smaller velopharyngeal cross sectional area. ${ }^{19-23}$ These craniofacial abnormalities have been reported in both white and Asian patients with OSAHS. ${ }^{24} 25$ Their measurement, however, requires the use of imaging modalities such as cephalometry and computed tomography, thus limiting their clinical utility. Most previous prediction models based on clinical variables have failed to include craniofacial measurements.

Few studies have reported the use of clinically derived craniofacial measurements in the prediction of OSA. Kushida and colleagues reported the development of a model based on BMI, NC, and oral cavity measurements. ${ }^{10}$ This model had a sensitivity of $97.6 \%$ and a specificity of $100 \%$ for the detection of OSA based on an AHI of $\geqslant 5$. The prevalence of OSA in the study by Kushida et al was $85 \%$ and analyses for different cutoff values for AHI were not performed. The results were strongly influenced by the large difference in BMI between patients with OSA and controls. This model required the performance of several complicated intraoral measurements using calipers, and it is questionable whether it would be suitable for routine clinical use. Friedman and colleagues examined the utility of various clinical features including upper airway measurements that predict a difficult endotracheal intubation. ${ }^{26}$ In a study of 172 patients they found that a modified MS, tonsil size and BMI (but not TMD) were predictive of OSA. In combination, these measurements accounted for $20 \%$ of the variance in respiratory disturbance index (RDI). Their analysis did not account for the potential interaction between BMI and the upper airway variables. Schellenberg and associates ${ }^{27}$ investigated several upper airway bony and soft tissue structural abnormalities for their ability to predict OSA after adjustment for important confounding factors. In a cohort of 420 subjects they measured the presence or absence of enlargement of the tonsils, uvula and tongue, low lying soft palate, retrognathia, overjet, and lateral pharyngeal narrowing. After adjusting for $\mathrm{BMI}$ and $\mathrm{NC}$, only lateral pharyngeal narrowing and tonsillar enlargement were associated with OSA. Skeletal features such as overjet and retrognathia were not significant predictors. A subgroup analysis of women yielded no significant associations, probably as a consequence of the small sample size. In that study one investigator performed all the morphometric measurements and intra and interobserver agreement were not assessed.

We chose simple clinical measurements of upper airway morphology such as the MS, TMD, and TMA because they are easy to perform. The MS has been used by anaesthetists for many years to help predict the degree of difficulty of endotracheal intubation. ${ }^{14}$ It reflects the amount of soft tissue in the posterior oropharynx relative to its skeletal 
constraints. The TMD represents the position of the chin relative to the thyroid cartilage and may be related to the length of the anterior cranial base. The MS and TMD have been shown to correlate with both difficult endotracheal intubation and OSA. ${ }^{26-28}$ The TMA has not been reported previously. To obtain an objective measurement of this feature of the craniofacial profile for the purpose of this study, we chose to determine the TMA from a lateral photograph of the head and neck obtained in a standardised fashion. We believe this angle reflects features of both obesity and abnormal craniofacial skeletal structure. Central obesity with accumulation of adipose tissue in the submental region will result in a large TMA. A short TMD or anterior cranial base will also result in a large TMA. Since the majority of patients with OSA have evidence of both obesity and craniofacial abnormalities, ${ }^{2}$ it is not surprising that TMA emerged as a significant predictor of OSA in our study. A high MS indicates a crowded posterior oropharynx which can be caused by obesity or craniofacial abnormality, or a combination of both. The predictive value of a large TMA and a high MS - after adjusting for ethnicity, BMI and NC, and for different AHI cut-off values for the definition OSAindicates a strong relationship between these two measurements and OSA. Both BMI and NC have previously been shown to correlate well with OSA, and both emerged as significant variables in our predictive equation, although less so than MS and TMA. Symptoms were not included in our predictive equation; a previous study found that they do not differentiate reliably between patients with and without OSA. ${ }^{29}$ Our results show that a simple clinical assessment of anthropometric features and craniofacial structure can help predict the presence of OSA.

Tsai and associates recently developed a decision rule for the diagnosis of OSA based on similar features of upper airway morphology including cricomental space (the perpendicular distance to the skin of the neck from the midpoint of a line joining the cricoid to the mentum); pharyngeal grade ( similar to the MS); and overbite..$^{30}$ Use of the decision rule allowed these investigators to confidently confirm or exclude OSA in $44 \%$ of patients. These results, together with our data, strongly suggest that clinical measurements from the craniofacial profile are good candidates for inclusion in the development of clinical prediction algorithms for the diagnosis of OSA and the prioritisation of patients for polysomnography. Our results extend the potential utility of these simple clinical measurements by demonstrating their applicability to patients of differing ethnicity and degrees of obesity.

We found that Asians had higher MS, shorter TMD and larger TMA, and tended to have more severe OSA than white subjects. Although the shorter TMD might suggest retrognathia, previous studies have shown that Asians have a more protruded maxilla and mandible. ${ }^{31}$ We suspect that the shorter TMD in Asians may be explained by a shorter anterior cranial base reflecting their smaller anteroposterior facial dimensions. ${ }^{32} \mathrm{Li}$ and associates compared two consecutive series of 50 Asian and 50 white men with OSA. ${ }^{6}$ When matched for obesity, Asians had more severe OSA than whites. After controlling for the severity of OSA, Asians were less obese than whites and had a more anteriorly positioned maxilla and mandible but a shorter anterior cranial base dimension $(\mathrm{p}<0.0001)$. Although the TMD did not differ between patients with OSA and normal controls in our study, the shorter TMD in Asians could contribute to a larger TMA and steeper thyromental plane in patients with OSA in this ethnic group. The higher MS and shorter TMD in Asians may reflect abnormalities of craniofacial anatomy that contribute to structural narrowing of the upper airway independent of obesity. The higher MS in Asians, reflecting more severe posterior oropharyngeal narrowing, might also explain why Asian men have more severe OSA than white men when matched for age and BMI. ${ }^{6}$ A few of our subjects (17\%) were women and, as shown in previous studies, they had smaller neck circumferences and less severe OSA than the men. The TMA and MS did not differ significantly between men and women.

Our study has certain limitations that may have an impact on the generalisability of our findings. There was a high prevalence of OSA in our sample, as defined by our a priori definition of $\mathrm{AHI} \geqslant 5$. We chose this definition to correspond with the generally accepted criteria proposed by the American Academy of Sleep Medicine. ${ }^{15}$ We performed post hoc analyses using different definitions for OSA which indicated that the predictive variables retained their utility over a wide range of cut-off values for AHI. Furthermore, discriminant analysis allows one to specify that prior probabilities are based on actual rather than equal group sizes, thus avoiding the problem of spuriously increased specificity. We studied only Asians and whites, so our results may not be applicable to other ethnic groups. There is a clear need for more data on the manifestations of sleep disordered breathing in different ethnic groups.

In conclusion, we have shown that risk factors for OSA such as obesity and craniofacial features that reflect structural narrowing of the upper airway are readily identified and can easily be incorporated into the routine physical examination. Patients with OSA of differing ethnicity and varying degrees of obesity have a crowded posterior oropharynx and a steep thyromental plane. Recognition of these abnormalities in the craniofacial profile should alert the physician to the possibility of OSA.

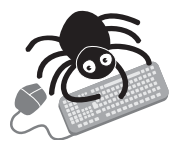

Details of the rationale and methodology for the statistical analysis are shown in the supplement available online at the Thorax website (http:// www.thoraxjnl.com/supplemental).

\section{Authors' affiliations}

B Lam, M S M Ip, University of Hong Kong, Hong Kong SAR, China E Tench, C F Ryan, Department of Medicine, University of British Columbia, Vancouver, BC, Canada

Supported by British Columbia Lung Association

\section{REFERENCES}

1 Isono S, Remmers JE, Tanaka A, et al. Anatomy of pharynx in patients with obstructive sleep apnea and in normal subjects. J Appl Physiol 1997;82:1319-26.

2 Ferguson KA, Takashi D, Lowe AA, et al. The relationship between obesity and craniofacial structure in obstructive sleep apnea. Chest 1995; 108:375-81.

3 Grunstein R, Wilcox I, Yang TS, et al. Snoring and sleep apnea in men: association with central obesity and hypertension. Int J Obesity 1993; 17:533-40

4 Stradling JR, Crosby JH. Predictors and prevalence of obstructive sleep apnea and snoring in 1001 middle-aged men. Thorax 1991;46:85-90.

5 Lowe AA, Fleetham JA, Adachi S, et al. Cephalometric and computed tomographic predictors of obstructive sleep apnea severity. Am J Orthod Dentofac Orthop 1995; 107:589-95.

6 Li KK, Kushida C, Powell NB, et al. Obstructive sleep apnea syndrome: a comparison between Far East Asian and white men. Laryngoscope 2000; 110:1689-93

7 Peker Y, Hedner J, Norum J, et al. Increased incidence of cardiovascular disease in middle-aged men with obstructive sleep apnea: a 7-year follow-up. Am J Respir Crit Care Med 2002;166:159-65.

8 Ross SD, Sheinhalt IA, Harrison KJ, et al. Systematic review and meta-analysis of the literature regarding the diagnosis of sleep apnea. Sleep 2000;23:519-32.

9 Rowley JA, Aboussouan LS, Badr S. The use of clinical prediction formulas in the evaluation of obstructive sleep apnea. Sleep 2000;23:929-37.

10 Kushida CA, Efron B, Guilleminault C. A predictive morphometric model for the obstructive sleep apnea syndrome. Ann Intern Med 1997;127:581-7.

11 Ryan CF, Love LL. Upper airway anatomy as a predictor of obstructive sleep apnea. Am J Respir Crit Care Med 1996;153:714A. 
12 Lam B, Ryan CF. Craniofacial profile predicts obstructive sleep apnea. Am J Respir Crit Care Med 2002; 165:407A.

13 Lundstrom F, Lundstrom A. Natural head position as a basis for cephalometric analysis. Am J Orthod Dentofac Orthop 1992;101:244-7.

14 Mallampati SR, Gatt SP, Gugino LD, et al. A clinical sign to predict difficult tracheal intubation: a perspective study. Can J Anaesth 1985;32:429-34.

15 Flemons WW. Sleep-related breathing disorders in adults: recommendations for syndrome definition and measurement techniques in clinical research. Sleep 1999:22:667-89.

16 Kapuniai LE, Andrew DJ, Crowell DH, et al. Identifying sleep apnea from selfreports. Sleep 1988;11:430-6.

17 Viner S, Szalai JP, Hoffstein V. Are history and physical examination a good screening test for sleep apnea? Ann Intern Med 1991;16:18-22.

18 Flemons WW, Whitelaw WA, Brant R, et al. Likelihood ratios for a sleep apnea clinical prediction rule. Am J Respir Crit Care Med 1994; 150:1279-85

19 Jamieson A, Guilleminault C, Partinen $M$, et al. Obstructive sleep apnea patients have craniomandibular abnormalities. Sleep 1986;9:469-77.

20 Lowe AA, Santamaria JD, Fleetham JA, et al. Facial morphology and obstructive sleep apnea. Am J Orthod Dentofac Orthop 1986;90:484-91.

21 Lowe AA, Ono T, Ferguson KA, et al. Cephalometric comparisons of craniofacial and upper airway structure by skeletal subtype and gender in patients with obstructive sleep apnea. Am J Orthod Dentofac Orthop 1996;110:653-64.

22 Maltais F, Carrier G, Cormier Y, et al. Cephalometric measurements in snorers, non-snorers, and patients with sleep apnea. Thorax $1991 ; 46: 419-23$
23 Zucconi M, Ferini-Strambi L, Palazzi S, et al. Habitual snoring with and without obstructive sleep apnoea: the importance of cephalometric variables. Thorax 1992;47:157-61.

24 Liu $Y$, Lowe AA, Zeng X, et al. Cephalometric comparisons between Chinese and Caucasian patients with obstructive sleep apnea. Am J Orthod Dentofac Orthop 2000; 117:579-85.

25 Ito D, Akashiba T, Yamamoto H, et al. Craniofacial abnormalities in Japanese patients with severe obstructive sleep apnea syndrome. Respirology 2001;6:157-61.

26 Friedman $M$, Tanyeri $H$, La Rosa $M$, et al. Clinical predictors of obstructive sleep apnea. Laryngoscope 1999;109:1901-7.

27 Schellenberg JB, Maislin G, Schwab RJ. Physical findings and the risk of obstructive sleep apnea. Am J Respir Crit Care Med 2000;162:740-8.

28 Hiremath AS, Hillman DR, James AL, et al. Relationship between difficult tracheal intubation and obstructive sleep apnoea. $\mathrm{Br} J$ Anaesth 1998:80:606-11.

29 Duran J, Esnaola S, Rubio R, et al. Obstructive sleep apnea-hypopnea and related clinical features in a population-based sample of subjects aged 30 to 70 yr. Am J Respir Crit Care Med 2001;163:685-9.

30 Tsai WH, Remmers JE, Brant $\mathrm{R}$, et al. A decision rule for diagnostic testing in obstructive sleep apnea. Am J Respir Crit Care Med 2003;167:1427-32.

31 Cooke MS, Wei SH. A comparative study of southern Chinese and British Caucasian cephalometric standards. Angle Orthod 1989;59:131-8.

32 Miyajima K, McNamara JA Jr, Kimura T, et al. Craniofacial structure of Japanese and European-American adults with normal occlusions and wellbalanced faces. Am J Orthod Dentofac Orthop 1996;1 10:431-8.

\section{bmjupdates+}

bmjupdates+ is a unique and free alerting service, designed to keep you up to date with the medical literature that is truly important to your practice.

bmjupdates+ will alert you to important new research and will provide you with the best new evidence concerning important advances in health care, tailored to your medical interests and time demands.

Where does the information come from?

bmjupdates+ applies an expert critical appraisal filter to over 100 top medical journals A panel of over 2000 physicians find the few 'must read' studies for each area of clinical interest

Sign up to receive your tailored email alerts, searching access and more...

www.bmjupdates.com 